\title{
Demand for and availability of specialist chemsex services in the UK: A cross- sectional survey of sexual health clinics
}

Wiggins H, Ogaz D, Mebrahtu H, Sullivan A, Bowden-Jones O, Field N, Hughes G

\section{BACKGROUND}

Chemsex has been defined as engaging in sexual activities while under the influence of specific recreational drugs (Crystal Methamphetamine, Mephedrone and $\mathrm{y}$-hydroxybutyrate $(\mathrm{GHB})^{1}$ ) taken before or during sex. This typically occurs between men who have sex with men (MSM) and is associated with high-risk sexual practices (group sex and condomless anal intercourse ${ }^{2,3}$ ) which in turn may result in increased transmission of sexually transmitted infections (STI's), enteric infections (notably Shigellosis ${ }^{3}$ ), Hepatitis $\mathrm{C}$ and HIV4

Multiple studies have demonstrated that MSM are more likely to have used recreational drugs then men who have sex exclusively with women ${ }^{2,5,6 . ~ I n ~ T h e ~ A s t r a ~ S t u d y ~}{ }^{2}$ half of the HIVpositive MSM studied reported recreational drug use in the previous 3 months: Among these nearly $20 \%$ reported condomless sex with partners of unknown HIV status and $32 \%$ group sex.

Targeted chemsex services have been developed in some Sexual Health Clinics (SHCs). 'The Chemsex Study' demonstrated, via qualitative interviews that in general, MSM felt comfortable discussing chemsex within Sexual Health Clinics (SHCs).

There are limited data available from the United Kingdom (UK) on the prevalence and frequency of chemsex in MSM and most are from SHCs in London or other large conurbations reflecting larger MSM populations in these areas. We undertook a survey of SHC healthcare workers (HCWs) to explore demand for and availability of chemsex services across the UK including in less urban areas, to inform service planning.

\section{METHOD}

\section{Survey development and implementation}

An online survey including questions on frequency of 'chemsex consultations' (patient reporting chemsex to medical staff during consultation), staff training, specialist services and clinic policies was developed using 'Select Survey'8 software with input from collaborating HCWs with clinical interest in chemsex. The survey was piloted by 2 SHC's and the members of British Association for Sexual Health and HIV (BASHH) National Audit Group (NAG) with no significant changes. (Appendix 1) The survey included two parts: Part 1 covered clinic policy and 2 clinical experiences and training. As a service development project a review by the research ethics committee was not required. 
The survey was distributed to all level $3^{9} \mathrm{UK}$ SHCs (complex service provision) via an email to clinical staff facilitated by trainee and NAG representatives. Non-responding clinics were sent reminders at one week.

Recipients were asked to distribute the survey to clinical staff including the most junior team members screening patients for STIs. To limit bias due to those with an interest in chemsex responding disproportionately only staff that worked on a specific day were invited to participate (except clinical leads who were asked to complete the survey regardless).

Clinical leads were asked to complete part 1. All respondents were asked to complete part 2.

\section{Data analysis}

Results were analysed by clinic. Where there were multiple responses per clinic, one response was selected for analysis, as follows:

(a) Any clinic with at least one respondent reporting chemsex consultations.

(b) Among the chemsex consultation clinics, the following hierarchy was applied, based on decreasing size of the respondent group: (i) nurses, (ii) doctors, (iii) HA's and (iv) 'other'. The exception was for the analysis of part 1 which was directed only at clinical leads.

For clinics in England, data were available from the national STI surveillance system (GUMCAD) ${ }^{10}$ and were used, along with data from the Office for National Statistics RuralUrban Classification for Local Authority Districts in England ${ }^{11}$, to stratify clinics into four geodemographic categories: (A) Urban conurbation, (B) urban with city/town, (C) urban with significant rural and (D) non- urban. Similar data were not available for non-English clinics which were therefore not stratified in the same way.

For clinic based analysis, Fisher's exact test with Bonferroni correction was used to examine associations between responses given by $\mathrm{HCW}$ and regional variations (i.e. rural/urban) of the participating clinics. STATA v.13.1 was used for analysis and $p$ values less than 0.01 were considered statistically significant (chosen after Bonferroni correction to address the fact that the probability of obtaining a spurious result increases with more tests taken).

Not all respondents answered each question leading to small variations in the denominators.

\section{RESULTS}

UK, RESPONDENT BASED DATA (results from all respondents)

Survey responses were received from 348 individuals from 56\% (150/270) of UK SHCs. The majority were from English clinics (90\%, 313/348). 1\% (3/348) were from Scottish, 1\% (4/348) Northern Irish (NI) and 3\% (10/348) Welsh clinics. This represents an overall clinic response rate of; $63 \%$ ( 135/214) for England, $8 \%$ ( 3/39) Scotland, 80\% (4/5) NI and 83\% for (10/12) 
Wales. For England, clinic contact details were available via GUMCAD. Similar information was not available outside of England and this may be reflected in lower response rates.

Using data about currently open English SHC's, the response rates by geo-demographic regions can be estimated as; $51 \%(40 / 78)$ for groups A, 53\% (41/68) B, 59\%(16/27) C and $47 \%(16 / 34)$ for $D$.

Most respondents worked in the following roles; Nurses (41\% 144/348), Doctors (24\% 84/348 consultants, junior doctors (5\% 16/348), associate specialists ( $7 \% 24 / 348)$, and HA's $(15 \%$, $52 / 348)$. There were a small number of respondents who worked in 'other' roles.

\section{CLINIC POLICY (Clinical leads only)}

With regards to clinic policy, data from $20 \%$ (55/270) of consultant clinical leads were available for analysis ( 1 clinical lead response per clinic selected). (48 England, 1 Scotland, 4 Wales, 2 N.I). For the majority of policy questions there were 44 responses. (44/55, 80\%). Due to the lower numbers, a geo-demographic analysis was not performed.

$11 \%(5 / 44)$ reported that a history of chemsex was routinely asked of all patients, $68 \%$ (30/44) selected patients and the remainder that it was not routinely asked.

Asked whether clinic policy requires documentation of a chemsex history in new patients, $69 \%(33 / 48)$ reported that it was not, $19 \%$ (9/48) that it was for selected patients and $13 \%$ $(6 / 48)$ for all patients. Where chemsex was reported, the documentation of associated harms (acute or chronic- physical, psychological, social or financial) was clinic policy in $10.4 \%(5 / 48)$ of clinics.

Where referral for ongoing management was required, 54\% (23/43) reported patient selfreferral, 33\% (14/43) a formal referral process, and 14\% (6/43) informal referral processes.

With regards to referral follow-up, $83 \%(39 / 47)$ did not have a process in place, $13 \%(6 / 47)$ did and 4\% (2/47) did not know.

\section{CLINC-BASED ANALYSIS}

\section{Demand for chemsex services (Table 1)}

$80 \%(103 / 129)$ of clinics reported ever seeing patients reporting chemsex and 50\% (65/129) that such consultations occurred at least monthly. These figures did not vary greatly across the UK (Fishers, $p=0.64$ ).

A demand for a local chemsex service was reported by $67 \%$ (87/129) of clinics.

\section{Chemsex Interventions (select all that apply)}


The most commonly reported interventions used were simple advice $(90 \%, 43 / 47)$, sign-post to NHS substance misuse services (53\%, 25/47), external needle exchanges $(55 \%, 26 / 47)$, brief-behavioural interventions $(53 \%, 25 / 47)$ and sign-post to online resources $(49 \%, 23 / 47)$. In-house chemsex and needle exchange interventions were available to $11 \%(5 / 47)$ of clinics, 3 of which were in London.

\section{Training}

$38 \%(44 / 114)$ of all respondents reported receiving chemsex training. This did not vary significantly by geo-demographic category in England, (Fishers, $p=0.016$, table 1)

Respondents from all but one clinic $(99 \%, 117 / 118)$ reported that there was a need for chemsex training but only $38 \%$ (44/117) reported previously having received training (Table 1). The most frequent areas of training requested were: Withdrawal (81\%, 96/118), terminology $(80 \%, 94 / 118)$, long-term harms of drug use $(75 \%, 89 / 118)$, drug interactions $(75 \%, 88 / 118,75 \%)$, acute harms $(69 \%, 82 / 118)$, assessment of 'problematic' chemsex $(66 \%$, $78 / 118)$ and history-taking $(63 \%, 74 / 118)$.

\section{Geo-demographic Analysis (England only, Table 1)}

In England, responding clinics were categorised in the following geodemographic regions: A urban conurbation $(35 \%, 40 / 113)$, B - urban with city/town $(36 \%, 41 / 113)$, C- urban with significant rural $(14 \%, 16 / 113)$, and D - non-urban (14\%, 16/113).

There was no significant difference in those reporting chemsex consultations or of the frequency of such consultations by geo-demographic region $(p=0.38$; Table 1$)$. Similarly there was no significant difference in training requirement $(p=0.016)$.

Response rates by geo-demographic region, to the question surrounding chemsex consultation frequency were $88-90 \%$ for groups $A, B$ and D and $69 \%$ for group C.

\section{DISCUSSION}

Our study suggests that patients reporting chemsex regularly present to SHCs throughout the UK, even in rural areas, and that while some have well-defined policies and care pathways in place, this is far from universal. Most clinics took a patient-targeted approach in establishing a chemsex history, lacked local chemsex specific services and had largely informal referral processes with no follow-up. The lack of a consistent approach across SHC's in England, Wales and Scotland is concerning, and clinical management of patients might be improved in some settings.

Our national, clinic-based survey provides much needed information about HCWs experiences of the demand for and availability of chemsex services, and the need for training about chemsex. More than half of all level three SHCs responded, such that we can be 
reasonably confident in generalising the findings in England and Wales, but caution is required when generalising to Scotland where response rates were lower.

There are important limitations of this work. Incomplete responses to some questions will have introduced inaccuracies. We cannot exclude the possibility that respondents were more likely to be HCWs with a particular interest in chemsex, resulting in over-estimation of the frequency of chemsex-related consultations. This was a self-reported survey and the results represent the views of the individuals responding and may not therefore accurately represent the whole clinic experience. Variability in respondents' roles will have influenced their knowledge and experience of, and expertise in, chemsex consultations in their clinic.

Appropriately targeted chemsex services may help to limit risk-taking behaviours and associated infections. One large London service demonstrated lower hepatitis $\mathrm{C}$ co-infection rates $(25 \%)$ within 2 years of implementing a chemsex service compared to other London clinics without such services (40\%). ${ }^{12}$ Addressing chemsex may have wider, positive impacts on patients' mental, social, financial and general health, as evidenced by a retrospective case review in one London service that revealed that half of MSM reporting chemsex perceived subsequent adverse consequences on their physical and mental health or career ${ }^{13}$.

The lack of chemsex specific services reported here may not be unreasonable given the variation in resources and service demand but an established clinical pathway for patient management should be available to all. The development of clinical networks (such as a hub and spoke model where the "hub" is the specialty's primary site and the "spokes" are connecting secondary sites serving that specialty) coupled with robust referral pathways might help to ensure that all patients have access to appropriate services regardless of their location. Such services should be non-judgmental, LGBT-friendly and able to assess and address the potential physical, psychological, social and financial harms associated with chemsex. Physical harms may include (amongst others) STI's or blood-borne viral infections, injecting site infections and withdrawal ${ }^{14}$.

Where chemsex is identified, clinicians need to be competent in assessing if this is problematic and in arranging appropriate intervention or referral. The development of a short screening tool could facilitate this. Universally accessible, up-to-date chemsex training is required, as evidenced by the $99 \%$ of respondents requesting further training. Two of the most requested training areas were about managing withdrawal and long-term harms related to drug use, issues traditionally managed in drugs and alcohol services. Collaboration, integration and possible co-location with such services for clinical delivery of chemsex services and training could be mutually beneficial. Further investigation is required to obtain a clearer picture of chemsex in the UK and a comprehensive survey involving patients directly is planned by PHE to help improve and direct services. Similar work in traditional drug and alcohol settings would also be beneficial. Routine questioning of all patients about chemsex would help to clarify the frequency of chemsex consultations in the UK and therefore guide 
service design and delivery. In England, PHE intend to introduce chemsex monitoring through routine national surveillance, and preliminary findings are promising ${ }^{11,15}$.

Our study suggests that the issue of chemsex is relevant to most SHC's across the country. It is critical that all patients participating in chemsex have access to local high quality, appropriate services to improve their health and well-being and avoid future harms. Improving training, developing clear care-pathways and collaboration between sexual health clinics could help deliver safe and effective treatment for this high-risk group of patients

\section{REFERENCES:}

1. McCall H. What is chemsex and why does it matter? The bmj 2015 Nov

2. Daskalopoulou $M$ et al. Recreational drug use, polydrug use and sexual behaviour in HIV-diagnosed men who have sex with men in the UK: results from the cross-sectional ASTRA study. The Lancet HIV 2014. October.

3. Gilbart VL et al. Sex, drugs and smart phone applications; findings from semi structured interviews with men who have sex with men diagnosed with Shigella flexneri 3a in England and Wales. Sex Transm Infect. 2015 Dec;91(8):598-602. doi: 10.1136/sextrans-2015-052014. Epub 2015 Apr 28.

4. Kall M, Shahmanesh M, Nardone A, Gilson R, Delpech V. Development of Positive Voices: the national survey of people living with HIV. P227. Third Joint Conference of the British HIV Association (BHIVA) with the British Association for Sexual Health and HIV (BASHH). Liverpool, UK; 1-4 April 2014.

5. Mercer $C$. The sexual health \& well-being of MSM in Britain: Evidence from the third National Survey of Sexual Attitudes \& Lifestyles (Natsal-3). British Association for Sexual Health and HIV Spring Conference 2015. June

6. Mohammed $\mathrm{H}$, et al on behalf of the GUMCADv3 Steering Group; Sexualised drug use in people attending sexual health clinics in England. Sex Transm Infect 2016;92:454. Epub 2016 Sept

7. Bourne et al; The Chemsex Study, Sigma Research Group; Int J Drug Policy.2015 Dec;26(12):1171-6.

8. Select Survey; Class apps; Selectsurvey.net

9. Parmar S. Department of Health. Integrated Sexual Health Services: National Service Specification- a suggested service specification for integrated sexual health services. Accessed via https://www.gov.uk/government/uploads/system/uploads/attachment_data/file/210726 /Service_Specification_with_covering_note.pdf (last accessed 26/7/2017)

10. Savage EJ, Mohammed H, Leong G, Duffell S, Hughes G. Improving surveillance of sexually transmitted infections using mandatory electronic clinical 
reporting: the genitourinary medicine clinic activity dataset, England, 2009 to 2013. Euro Surveill. 2014;19(48):pii=20981

11. Office of National Statistics. 2011 rural/urban classification. Accessed via http://webarchive.nationalarchives.gov.uk/20160105160709/http://www.ons.gov.uk/on s/guide-method/geography/products/area-classifications/2011-rural-urban/index.html (accessed 30th January 2017)

12. 56 Dean St and Hepatitis C Trust. Chemsex and hepatitis C: A guide for healthcare providers. December 2014. Accessed via http://www.chelwest.nhs.uk/services/hiv-sexual-health/professionals/links/ChemSexHep-C-Guide.pdf (last accessed 30th Jan 2017)

13. Lee MJ et al. Chemsex and the city: sexualized substance use in gay bisexual and other men who have sex with men. Accessed via https://www.bashh.org/media/1287/o11-ming-lee.pdf (last accessed 30th Jan 2017) 14. BASHH statement on club (recreational) drug use. Accessed via https://www.bashh.org/documents/BASHH\%20Statement\%20on\%20'club'\%20(recrea tional)\%20drug\%20use.pdf Last accessed ( $8^{\text {th }}$ November 2017) Mohammed $\mathrm{H}$ et al. Sexualised drug use in people attending sexual health clinics in England. Sexually Transmitted Infections. 2016 Sept;92(6):454

15. Mohammed $\mathrm{H}$ et al. Sexualised drug use in people attending sexual health clinics in England. Sexually Transmitted Infections. 2016 Sept;92(6):454 